\title{
Frustating use of the Laplace transform for the quantum states of a particle in a box
}

\author{
Antonio S. de Castro*1@ \\ ${ }^{1}$ Universidade Estadual Paulista "Júlio de Mesquita Filho", Departamento de Física e Química, Guaratinguetá, SP, Brasil
}

Received on March 1, 2020. Accepted on March 30, 2020.

\begin{abstract}
In a recent paper diffused in the literature, the quantum problem of a particle in a infinite square well potential was claimed to be solved via Laplace transform. Surprisingly, the correct solutions were found for an eigenvalue problem with variable defined on a finite range. As a matter of fact, the authors have misused the Laplace transform of the time-independent Schrödinger equation, and they have also used incorrectly the inversion process. In the present paper it is pointed out carefully and clearly those mistakes that might occur with the use of inappropriate methods for a given eigenvalue problem.
\end{abstract}

Keywords: Infinite square-well potential, Particle in a box, Laplace transform.

\section{Introduction}

Integral transform methods are useful for solving differential equations when they can convert the original equation into a simpler equation. The inversion of the transform for reconstructing the original function may be a rather complicated calculation. If this is the case and one does not find the integral in tables, the method will be worthless. The Laplace transform method was used at the first years of quantum mechanics by Schrödinger into the discussion of radial eigenfunction of the hydrogen atom 1], and more than forty years later Englefield approached the Schrödinger equation with the Coulomb, oscillator, exponential and Yamaguchi potentials [2]. More than twenty years went by and the hydrogen atom was again examined with the Laplace transform method [3]. For some years now the $1 / x$ [4], Morse [5], $N$-dimensional harmonic oscillator [6], pseudoharmonic and Mie-type [7], and double Dirac delta 8 potentials have been solved for the Laplace transform. The one-dimensional quantum harmonic oscillator problem has been revisited [9], and it has been shown that the bound-state solutions of the Schrödinger equation whose eigenfunctions are expressed in terms of particular solutions of the confluent hypergeometric equation can be obtained by using the Laplace transform of the confluent hypergeometric equation [10]. In a recent paper diffused in the literature [11], the authors claimed to solve the quantum problem of a particle in a infinite square well potential via Laplace transform. The appropriate method to solve a given eigenvalue problem depends on the character of the boundary conditions, and for the Laplace transform method the range of variation of the variable is defined on the infinite half line. It is surprising that the authors of Ref. 11] found the

*Correspondence email address: antonio.castro@unesp.br correct solutions for an eigenvalue problem with variable defined on a finite range. As a matter of fact, they have misused the Laplace transform of the time-independent Schrödinger equation, and they have also used incorrectly the inversion process. The purpose of the present paper is to point out carefully and clearly those mistakes that might occur with the use of inappropriate methods for a given eigenvalue problem.

\section{The infinite square well}

One of the easiest problems in quantum mechanics, the particle in a box, so called because the infinite square well confines the particle between impenetrable walls, is a problem usually explored in the introductory textbooks on quantum mechanics (see, e.g. [12]- 223]), keeping strictly to the straight and narrow resolution of the timeindependent Schrödinger equation

$$
-\frac{\hbar^{2}}{2 m} \frac{d^{2} \psi_{E}(x)}{d x^{2}}+V(x) \psi_{E}(x)=E \psi_{E}(x),
$$

with

$$
V(x)=\left\{\begin{array}{l}
0, \quad 0 \leq x \leq L \\
\infty, \quad x<0 \quad \text { and } \quad x>L,
\end{array}\right.
$$

in such a way that

$$
\left(\frac{d^{2}}{d x^{2}}+k^{2}\right) \psi_{E}(x)=0, \quad 0 \leq x \leq L
$$

where

$$
k=\sqrt{\frac{2 m E}{\hbar^{2}}},
$$

and

$$
\psi_{E}(x)=0, \quad x<0 \quad \text { and } \quad x>L .
$$


In addition, homogeneous Dirichlet boundary conditions are imposed on the confining walls, viz. $\psi_{E}(0)=\psi_{E}(L)=$ 0 . Therefore, the eigenfunction $\psi_{E}(x)$ can be compactly written as

$$
\psi_{E}(x)=\theta(x) \theta(L-x) f_{E}(x),
$$

where $\theta(x)$ is is the step function

$$
\theta(x)= \begin{cases}1, & x>0 \\ 0, & x<0\end{cases}
$$

and $f_{E}(x)$ satisfies the equation

$$
\left(\frac{d^{2}}{d x^{2}}+k^{2}\right) f_{E}(x)=0, \quad 0 \leq x \leq L,
$$

subject to the homogeneous Dirichlet boundary conditions $f_{E}(0)=f_{E}(L)=0$.

\section{Laplace transform and a few of its properties}

The Laplace transform of $\phi(t)$ is defined by (see, e.g. 24])

$$
\mathcal{L}\{\phi(t)\}=F(s)=\int_{0}^{\infty} d t e^{-s t} \phi(t) .
$$

The Laplace transform has the linearity property, meaning that if $\phi_{1}(t)$ and $\phi_{2}(t)$ are two functions whose Laplace transform exists,

$$
\mathcal{L}\left\{\lambda_{1} \phi_{1}(t)+\lambda_{2} \phi_{2}(t)\right\}=\lambda_{1} \mathcal{L}\left\{\phi_{1}(t)\right\}+\lambda_{2} \mathcal{L}\left\{\phi_{2}(t)\right\},
$$

where $\lambda_{1}$ and $\lambda_{2}$ are constants. If there is some constant $\sigma \in \mathbb{R}$ such that

$$
\left|e^{-\sigma t} \phi(t)\right| \leq M
$$

for sufficiently large $t$, the integral in equation 9 will exist for $\operatorname{Re} s>\sigma$ and $\phi(t)$ is said to be of exponential order. On the other hand, if $\phi(t) \rightarrow t^{\alpha}$ as $t \rightarrow 0$ the existence of the Laplace transform demands $\operatorname{Re} \alpha>-1$ and $\operatorname{Re} s>0$ because

$$
\mathcal{L}\left\{\frac{t^{\alpha}}{\Gamma(\alpha+1)}\right\}=\frac{1}{s^{\alpha+1}}, \quad \operatorname{Re} \alpha>-1, \quad \operatorname{Re} s>0 .
$$

where $\Gamma(z)$ is the gamma function. One should note the shifting property

$$
\mathcal{L}\{\theta(t-a) \phi(t-a)\}=e^{-s a} \mathcal{L}\{\phi(t)\}, \quad a>0 .
$$

An example of Laplace transform is

$$
\mathcal{L}\{\sin k t\}=\frac{k}{s^{2}+k^{2}}, \quad \operatorname{Re} s>0 .
$$

Derivative properties involving the Laplace transform are convenient for solving differential equations:

$$
\begin{gathered}
\mathcal{L}\left\{\frac{d \phi(t)}{d t}\right\}=s \mathcal{L}\{\phi(t)\}-\left.\phi(t)\right|_{t=0} \\
\mathcal{L}\left\{\frac{d^{2} \phi(t)}{d t^{2}}\right\}=s^{2} \mathcal{L}\{\phi(t)\}-\left.s \phi(t)\right|_{t=0}-\left.\frac{d \phi(t)}{d t}\right|_{t=0} .
\end{gathered}
$$

\section{Trying to solve the problem}

The eigenfunction parameterized as in equation (6) has limiting behaviors that make it possible the existence of the Laplace transform. This Laplace transform of $\psi_{E}(x)$ can be written as

$$
\mathcal{L}\left\{\psi_{E}(x)\right\}=\int_{0}^{L} d x e^{-s x} f_{E}(x),
$$

with the following shifting property

$$
\mathcal{L}\left\{\theta(x-L) \psi_{E}(x-L)\right\}=0 \text {. }
$$

For $\psi_{E}(x)=\sin k x$, for example, one obtains

$$
\mathcal{L}\left\{\psi_{E}(x)\right\}=\frac{k}{s^{2}+k^{2}}-e^{-s L} \frac{s \sin k L+k \cos k L}{s^{2}+k^{2}} .
$$

Furthermore, $\mathcal{L}\left\{\psi_{E}(x)\right\}$ has the following derivative properties

$$
\begin{aligned}
& \mathcal{L}\left\{\frac{d \psi_{E}(x)}{d x}\right\}=s \mathcal{L}\left\{\psi_{E}(x)\right\}-\left.f_{E}(x)\right|_{x=0} \\
& +\left.e^{-s L} f_{E}(x)\right|_{x=L} \\
& \mathcal{L}\left\{\frac{d^{2} \psi_{E}(x)}{d x^{2}}\right\}=s^{2} \mathcal{L}\left\{\psi_{E}(x)\right\}-\left.s f_{E}(x)\right|_{x=0} \\
& -\left.\frac{d f_{E}(x)}{d x}\right|_{x=0}+e^{-s L}\left[\left.s f_{E}(x)\right|_{x=L}\right. \\
& \left.+\left.\frac{d f_{E}(x)}{d x}\right|_{x=L}\right] .
\end{aligned}
$$

Using the homogeneous Dirichlet boundary conditions imposed on $f_{E}(x)$ at the walls, one obtains

$$
\begin{aligned}
\mathcal{L}\left\{\frac{d \psi_{E}(x)}{d x}\right\} & =s \mathcal{L}\left\{\psi_{E}(x)\right\} \\
\mathcal{L}\left\{\frac{d^{2} \psi_{E}(x)}{d x^{2}}\right\} & =s^{2} \mathcal{L}\left\{\psi_{E}(x)\right\} \\
& -\left.\frac{d f_{E}(x)}{d x}\right|_{x=0}+\left.e^{-s L} \frac{d f_{E}(x)}{d x}\right|_{x=L} .
\end{aligned}
$$

The Laplace transform of equation (8), using the linearity and the derivative properties of the Laplace transform, establishes a mapping of the second-order differential equation for $f_{E}(x)$ and into a first-degree algebraic equation for $\mathcal{L}\left\{\psi_{E}(x)\right\}$ :

$$
\begin{aligned}
& \left(s^{2}+k^{2}\right) \mathcal{L}\left\{\psi_{E}(x)\right\}-\left.\frac{d f_{E}(x)}{d x}\right|_{x=0} \\
& +\left.\frac{d f_{E}(x)}{d x}\right|_{x=L} e^{-s L}=0
\end{aligned}
$$


which is certainly simpler than the original second-order differential equation, and has as a solution

$$
\begin{aligned}
& \mathcal{L}\left\{\psi_{E}(x)\right\}=\frac{\left.\frac{d f_{E}(x)}{d x}\right|_{x=0}}{k} \frac{k}{s^{2}+k^{2}}- \\
& \frac{\left.\frac{d f_{E}(x)}{d x}\right|_{x=L}}{k} e^{-s L} \frac{k}{s^{2}+k^{2}} .
\end{aligned}
$$

In view of equation (18), one can not identify the inverse Laplace transform of $k /\left(s^{2}+k^{2}\right)$ with $\sin k x$, and the second term on the right-hand side of equation (23) presents a more challenging inversion process. All the difficulty that prevents us of doing the inversion is due to the boundary term proportional to $e^{-s L}$ which is missing in Ref. [11], and vanishes only when $\operatorname{Re} s>0$ and $L \rightarrow \infty$.

\section{Final remarks}

It has been clearly shown that the approaching of the quantum problem of a particle in a infinite square well potential via Laplace transform claimed by the authors of Ref. 11 is not reliable and consistent. The appropriate method to solve a given eigenvalue problem depends on the character of the boundary conditions. For the Laplace transform, and also for the Fourier transform, the range of variation of the variable is infinite whereas for the particle in a box the range is finite. An appropriate alternative way for approaching the particle in a box would be via Fourier series which are useful for representing functions defined over a limited range (or over an infinite range if the function is periodic).

\section{Acknowledgement}

This work was supported in part by means of funds provided by Conselho Nacional de Desenvolvimento Científico e Tecnológico (CNPq), Brazil, Grant No. 09126/2019-3 (PQ).

\section{References}

[1] E. Schrödinger, Ann. Physik 384, 361 (1926).

[2] M.J. Englefield, J. Austr. Math. Soc. 8, 557 (1968).

[3] R.A. Swainson and G.W.F. Drake, J. Phys. A 24, 79 (1991).

[4] Y. Ran, L. Xue, S. Hu and R.K. Su, J. Phys. A 33, 9265 (2000).

[5] G. Chen, Phys. Lett. A 326, 55 (2004).

[6] G. Chen, Chin. Phys. 14, 1075 (2005).

[7] A. Arda and R. Server, J. Math. Chem. 50, 971 (2012).

[8] A.S. de Castro, Rev. Bras. Ens. Fis. 34, 4301 (2012).

[9] D.R.M. Pimentel and A.S de Castro, Eur. J. Phys. 34, 199 (2013).

[10] P.H.F. Nogueira, A.S. de Castro and D.R.M. Pimentel, J. Math. Chem. 54, 1287 (2016).

[11] R. Gupta, R. Gupta and D. Verma, IJITEE 8, 6 (2019).
[12] D.J. Griffiths, Introduction to Quantum Mechanics (Prentice Hall, New Jersey, 1955).

[13] A. Messiah, Mecanique Quantique (Dunod, Paris, 1964), v. 1.

[14] A.S. Davydov, Quantum Mechanics (Pergamon Press, Oxford, 1965).

[15] L.I. Schiff, Quantum Mechanics (McGraw-Hill, New York, 1968).

[16] G. Baym, Lectures in Quantum Mechanics (Benjamin, New York, 1969).

[17] E. Merzbacher, Quantum Mechanics (Wiley, New York, 1970).

[18] S. Flügge, Practical Quantum Mechanics (SpringerVerlag, Berlin, 1971), v. I.

[19] R. Eisberg and R. Resnick, Quantum Physics of Atoms, Molecules, Solids, Nuclei and Particles (Wiley, New York, 1974).

[20] C. Cohen-Tannoudji, B. Diu and F. Laloë, Quantum Mechanics (Hermann, Paris, 1977), v. 1.

[21] W. Greiner, Quantum Mechanics: An Introduction (Berlin, Springer-Verlag, 1989).

[22] R. Shankar, Principles of Quantum Mechanics (Plenum Press, New York, 1994).

[23] R.W. Robinett, Quantum Mechanics (Oxford University Press, Oxford, 2006), $2^{\mathrm{a}}$ ed.

[24] G. Doetsch, Introduction to the Theory and Application of the Laplace Transformation (Springer, New York, 1974). 